\title{
EVERY LOCALLY COMPACT MAP GROUP IS UNIMODULAR
}

\section{H. LEPTIN AND L. ROBERTSON}

A topological group $G$ is Maximally Almost Periodic (MAP), if $G$ admits a continuous monomorphism in to a compact Hausdorff group, for references see e.g. [6].

Here we consider only locally compact MAP groups. For such groups we will prove the title of this note by first reducing the problem to the totally disconnected case, and then applying a general result on measure-preserving properties of automorphisms of totally disconnected groups. The authors wish to thank S. Grosser, M. Moskowitz, and T. Wilcox for interesting conversations on this topic.

Proposition 1. Suppose $G$ is maximally almost periodic, and suppose that $H$ is a normal subgroup of $G$ which is either compact or else equals the center of $G$. Then $G / H$ is maximally almost periodic.

Proof. Let $\pi$ denote the homomorphism from $G$ to the Bohr compactification $b G$ of $G$ (see [3, Chapter 8]). If $H$ is compact, then $\pi(H)$ is closed and normal in $b G$, and $b G / \pi(H)$ is a compact group. The fact that the quotient group mapping from $G$ to $G / H$ is an open mapping then shows that $G / H$ has a continuous homomorphism into the compact group $b G / \pi(H)$, and this homomorphism is injective because $\pi(x) \notin \pi(H)$ when $x \notin H$. If $H=Z(G)$ is the center of $G$, then we may likewise imbed $G / H$ into $b(G) / Z(b(G))$, and the same arguments apply because $x$ is in the center of $G$ iff $\pi(x)$ is in the center of $b(G)$.

Proposition 2. Let $H$ be a normal subgroup of $G$ which is either a central subgroup or a compact subgroup. Then $G$ is unimodular if and only if $G / H$ is unimodular.

Proof. Let mod $i_{x}$ denote the factor by which $H$-measure is multiplied when $x$ acts as a conjugation operator $i_{x}$ on $H$. Then $\bmod i_{x}=1$ if $H$ is central (because $i_{x}$ is trivial) and $\bmod i_{x}=1$ if $H$ is compact (because every homeomorphic automorphism of a compact group is measure-preserving). Letting $G / H=Q=\pi(G)$, and letting $\delta$ denote the modular function, the result follows from the equation $\delta_{G}(x)$ $=\delta_{Q}(\pi(x)) \bmod i_{x}$ of [2, Proposition 11, Corollary, p. 61].

Proposition 3. Let $H$ be a subgroup of $G$ such that $H$ has finite index in $G$. Then $H$ is MAP (respectively unimodular) if and only if $G$ is $M A P$ (respectively unimodular).

Received by the editors May 20, 1967. 
Proof. The assertion about MAP is well known, and is proven in [5, Lemma 4, p. 124]. An alternative proof using the theory of induced representations is available in [8, Theorem 1.5.4, p. 18]. As for unimodularity, let us first consider the case of $N$ a normal subgroup with finite index in $G$. Then using the fact that $\delta_{G}(n)=\delta_{N}(n)$ for $n \in N,[2$, Theorem 3, Corollary 2, p. 59], we see that $G$ fails to be unimodular if $N$ fails to be unimodular. If $\delta_{G}$ is trivial on $N$, and if $N$ has finite index in $G$, then $\delta_{G}$ maps on to a finite subgroup of $R^{+}$. Thus unimodularity of $N$ implies unimodularity of $G$. For the general case of $H$ with finite index in $G$, we let $N$ denote the intersection of the conjugates of $H$. Then $N$ is normal in $G$ with finite index, and $\delta_{N}(n)$ $=\delta_{H}(n)=\delta_{G}(n)$ for $n \in N$. Thus the above shows that $H$ is unimodular iff $N$ is unimodular, and $N$ is unimodular iff $G$ is unimodular.

Proposition 4. If there is an MAP group which fails to be unimodular, then there is a nonunimodular MAP group which is a central extension of the vector group $R^{n}$ by a totally disconnected group.

Proof. Let $G$ be MAP with identity component $G_{0}$. Then the Freudenthal-Weil theorem [7, pp. 127-129] shows that $G_{0}=K+R^{n}$ has a projection $\pi$ onto $R^{n}$. Applying an inner automorphism of $G$ to obtain an automorphism $\alpha$ of $G_{0}$, we have that $\pi(\alpha(K))$ is a compact subgroup of $R^{n}$, and so $\alpha(K)=K$. Thus $K$ is a normal subgroup of $G$, and we may apply Propositions 1 and 2 to get a nonunimodular MAP group $G_{1}$ which has $R^{n}$ as its identity component. It follows from [8, Theorem 4.5, p. 63] that $G_{1}$ is a finite extension of a central extension of $R^{n}$. (This can be seen by studying the orbits in the dual of $R^{n}$ under the action of $G_{1}$, where $G_{1}$ acts canonically by inner automorphisms on $R^{n}$ as in [1]. First observe that the union of the finite orbits of the dual group $R^{n}$ is a dense subgroup $S$. Picking a basis of $R^{n}$ from $S$, we see that $G_{1}$ acts as a finite group of linear endomorphisms of $R^{n}$. It follows then that the kernel of the canonical homomorphism must be a normal subgroup with finite index.) Having obtained a normal subgroup with finite index which is a central extension of the identity component of $G_{1}$, it follows from Proposition 3 that there is a nonunimodular MAP group which is a central extension of $R^{n}$ by a totally disconnected group.

Proposition 5. If there is a nonunimodular MAP group, then there is a nonunimodular MAP group which is totally disconnected.

Proof. Using Proposition 4 to obtain a nonunimodular MAP group $G$ such that $G_{0}=R^{n}$ and $R^{n} \subset Z(G)$, it follows from Propositions 1 and 2 that $G / Z(G)$ is a nonunimodular MAP group which is totally disconnected. (Note that the center $Z(G)$ is a proper subgroup of $G$, 
since $G$ is nonunimodular. Moreover, there is no problem if $G_{0}=R^{n}$ with $n=0$.)

Now let $G$ be a totally disconnected locally compact group.

Proposition 6. G is MAP if and only if there is a family $\Re$ of open normal subgroups $N$ of $G$ such that all $G / N$ are (discrete) $M A P$ and $\cap_{N \in \mathfrak{I}} N=(e)$.

Clearly the condition is sufficient. Now let $\phi$ be a continuous homomorphism of $G$ into a compact connected Lie group $K$ and let $N$ be the kernel of $\phi$. The open subgroups form a neighborhood base at the identity in $G, \phi$ is continuous and $K$ does not have arbitrarily small subgroups (see $[4, \S 2.16$, p. 73$]$ ); hence $N$ contains an open subgroup so $N$ must be open. Evidently $G / N$ is MAP and because $G$ is MAP the $\phi$ 's separate points, i.e. $\cap N=(e)$.

THEOREM 1. Let $G$ be a locally compact group, $\alpha$ a homeomorphic automorphism of $G$ and $\mathfrak{N}$ a family of open subgroups of $G$. If $\cap_{N \in \mathfrak{r}} N$ $=(e)$ and $\alpha(N)=N$ for all $N \in \mathfrak{N}$, then $\alpha$ is Haar measure-preserving.

Proof. Taking all intersections of finitely many of the $N \in \mathfrak{N}$, we may assume that $\mathfrak{X}$ is directed with respect to inclusion. For a subset $X$ and a subgroup $N$ in $G$ let $|X|{ }_{N}$ denote the number of different cosets $x N, x \in X$, in $G$. If $X$ is Haar-measurable, let $|X|$ be the Haarmeasure of $X$. There exists an $a>0$ such that $|\alpha(X)|=a|X|$ for all measurable sets $X \subset G$. Furthermore observe that $|\alpha(X)|_{N}=|X|_{N}$ if $\alpha(N)=N$, especially for all $N \in \Re$. Now let $U$ and $V$ be compact open subgroups of $G$ with $V \subset U$ (observe that our hypotheses imply that $G$ is totally disconnected!). Let $U: V$ be the (finite) index of $V$ in $U$.

Lemma. There exists $N_{0} \in \mathfrak{N}$ such that $U: V=|U|_{N} /|V|_{N}$ if $N \subset N_{0}$, $N \in \Re$.

Proof of the Lemma. $U$ is a compact and $\bigcap_{N \in \mathfrak{M}}(N \cap U)=(e)$, thus every neighborhood of $e$ in $U$ contains a subgroup $N \cap U$ (because the sets $N \cap U$ cannot have the finite intersection property on any closed subset of $U$ which misses $e$ ). Choose $N_{0}$ in $\Re$ with $N_{0} \cap U \subset V$. Then for $N \in \Re, N \subset N_{0}$, we have $N \cap U=N \cap V$. Now the mapping $z N \rightarrow z(N \cap U), z \in U$, is one-to-one from the set of all cosets $z N, z \in U$, on to the left coset space of $N \cap U$ in $U$. Hence $|U|_{N}=U:(N \cap U)$ and similarly $|V|_{N}=V:(N \cap V)$. This implies

$$
\frac{|U|_{N}}{|V|_{N}}=\frac{U:(N \cap V)}{V:(N \cap V)}=U: V
$$

if $N \subset N_{0}$. 
Now let $W_{0}$ be a compact open subgroup and normalize Haar measure such that $\left|W_{0}\right|=1$. Apply the lemma to $U$ and $U \cap W_{0}$ and to $W_{0}$ and $U \cap W_{0}$ : there exists $N \in \Re$ with

$$
\frac{U: U \cap W_{0}}{W_{0}: U \cap W_{0}}=\frac{|U|_{N} /\left|U \cap W_{0}\right|_{N}}{\left|W_{0}\right|_{N} /\left|U \cap W_{0}\right|_{N}}=\frac{|U|_{N}}{\left|W_{0}\right|_{N}} .
$$

For open subgroups $A$ and $B$ with $B C A$ we always have $|A|$ $=(A: B)|B|$. Hence the quotients in the last equation are equal to $|U| /\left|W_{0}\right|=|U| ;$ which yields $|U|=|U|_{N} /\left|W_{0}\right|_{N}$. This finally gives

$$
a|U|=|\alpha(U)|=\frac{|\alpha(U)|_{N}}{\left|W_{0}\right|_{N}}=\frac{|U|_{N}}{\left|W_{0}\right|_{N}}=|U|>0,
$$

and so $a=1$.

COROllary. If $G$ has a family $\mathfrak{x}$ of open normal subgroups with $\cap_{N \in \mathfrak{X}} N=(e)$, then $G$ is unimodular.

Proof. Take for $\alpha$ an inner automorphism and apply Theorem 1.

Now we are ready to prove

Theorem 2. A locally compact MAP group is unimodular.

Proof. Proposition 5 tells us that we need only consider totally disconnected groups. For this case Proposition 6 shows that a MAP group $G$ has a system of open normal subgroups intersecting in $(e)$. Therefore the corollary of Theorem 1 applies and proves that $G$ is unimodular.

\section{Bibliography}

1. R. J. Blattner, Group extension representations and the structure space, Pacific J. Math. 15 (1965), 1101-1113.

2. N. Bourbaki, Éléments de mathématique, Fasc. XXIX, Livre VI. Intégration. Chapitre 7, Mesure de Haar, Actualités Sci. Indust. No. 1306, Hermann, Paris, 1963.

3. L. H. Loomis, An introduction to abstract harmonic analysis, Van Nostrand, New York, 1953.

4. D. Montgomery and L. Zippin, Topological transformation groups, Interscience, New York, 1955.

5. S. Murakami, Remarks on the structure of maximally almost periodic groups, Osaka J. Math. 2 (1950), 119-129.

6. J. von Neumann, Almost periodic functions in a group, Trans. Amer. Math. Soc. 36 (1934), 445-492.

7. A. Weil, L'intégration dans les groupes topologiques et ses applications, 2nd ed., Actualités Sci. Indust. No. 1145, Hermann, Paris, 1965.

8. T. Wilcox, On the structure of maximally almost periodic groups, Thesis, Univ. of Washington, Seattle, 1967.

Institut für Angewandte Mathematik, Heidelberg, Germany, ANd UNIVERSITY OF WASHINGTON 